\title{
DETERMINACIÓN DEL FACTOR “ $\eta ”$ PLÁSTICO PARA PROBETAS NO NORMALIZADAS TIPO SE(T)
}

\section{Iván Aguirre, I. Ferreira y Francisco Aguirre}

\section{RESUMEN}

La determinación de los parámetros de la mecánica de fractura Elástico-Plástica a partir de probetas no normalizadas tipo tracción con entalla lateral Single Edge notch Tensile, SE(T), viene ganando importancia. Trabajos publicados recientemente muestran que este tipo de probetas tiene condiciones de restricción del flujo plástico en la punta de la fisura muy semejantes a lo que ocurre en poliductos que presentan fisuras. Para probetas no normalizadas del tipo $\mathrm{SE}(\mathrm{T})$ existe poca información sistematizada relatada en la bibliografía de cómo determinar este factor. En este trabajo se determina el factor $\eta$ plástico para probetas $\mathrm{SE}(\mathrm{T})$ a partir de datos obtenidos del método analítico EPRI y por modelado numérico basado en el método de elementos finitos para mediciones del desplazamiento de abertura en la boca de la fisura CMOD y en la línea de carga LLD. La validación de las metodologías propuestas fue realizada por el modelado de probetas normalizadas tipo $\mathrm{SE}(\mathrm{B})$, y los resultados comparados con los encontrados en la literatura. Los resultados del factor $\eta$ plástico para probetas $\mathrm{SE}(\mathrm{T})$ calculados numéricamente en este trabajo, son comparados con los obtenidos a través de datos proporcionados por el método analítico EPRI, en ambos casos se obtuvo valores del factor $\eta$ plástico cercanos a uno para mediciones de CMOD, siendo estos valores casi independientes del tamaño relativo de la fisura y del exponente de acritud. Finalmente, este resultado fue utilizado para la determinación experimental de la integral J con probetas SE(T) fabricadas de acero API 5L X65, que es un acero utilizado en la fabricación de tuberías industriales.

Palabras Clave: Factor H Plástico, SE(T), Acero API 5L X65, Tenacidad de Fractura. 